\title{
High Efficacy in Hyperthermia-associated with Polyphosphate Magnetic Nanoparticles for Oral Cancer Treatment
}

\section{Candido NM¹ ${ }^{1}$, Calmon MF${ }^{1}$, Taboga SR ${ }^{1}$, Bonilha $\mathrm{JL}^{2}$, dos Santos MC ${ }^{3}$, Lima ECD ${ }^{3}$, Sousa CRS ${ }^{4}$, Lacava ZGM ${ }^{4}$ and Rahal ${ }^{1 *}$}

${ }^{1}$ São Paulo State University “Júlio de Mesquita Filho”, UNESP, Institute of Bioscience, Language \& Literature and Exact Science, Department of Biology, 15054-000, São José do Rio Preto, SP, Brazil

${ }^{2}$ Medical School of São José do Rio Preto, FAMERP, Department of Pathology and Forensic Medicine, 15090-000, São José do Rio Preto, SP, Brazil

${ }^{3}$ Federal University of Goiás, UFG, Institute of Chemistry, P.C. 131, 74001-970, Goiânia, GO, Brazil

${ }^{4}$ University of Brasilia, UnB, Institute of Biological Sciences, CNANO, GEM, 70910-900, Brasilia, DF, Brazil

\begin{abstract}
Nanotherapy applied to cancer treatment is constantly evolving, and new approaches to current techniques, such as magnetohyperthermia, are being implemented to solve and minimize the limitations of conventional therapeutic strategies. The purpose of this study was to investigate the action of polyphosphate-coated maghemite nanoparticles (MNPs) on oral squamous cell carcinoma. Human oral cancer cells (UM-SCC14A) were incubated with MNPs at various concentrations and subjected to cell proliferation tests (MTT), apoptosis assays and transmission electron image analysis. Viability and apoptotic events were time and dose dependent. These in vitro tests showed that at the intermediate concentration tested there is no significant toxicity, as confirmed by transmission electron microscopy. For this reason this MNPs concentration was chosen for the subsequent in vivo tests. Oral tumor induction was performed by applying the carcinogen DMBA to Syrian hamsters. Animals were then treated by magnetohyperthermia using MNPs. No signs of general clinical symptoms of toxicity or abnormal behavioral reactions were observed. However, animals treated with MNPs and exposed to the alternating magnetic field in the hyperthermia procedure exhibited a significant and time dependent cancer regression, as confirmed by histopathological analyses and immunohistochemistry. Actually, in quantitative terms of the magnetotherapy efficacy involving these polyphosphate-coated MNPs, 100\% recovery (12/12) was observed in the oral cancer tumor bearing Syrian hamsters seven days after the treatment with the magnetohyperthermia procedure. Data supports the suggestion that the MNPs-mediated hyperthermia represents a promising strategy for the treatment of oral cancer.
\end{abstract}

Keywords: Oral squamous cell carcinoma; UM-SCC14A; Syrian hamster; Magnetic nanoparticles coated with polyphosphate; Magnetohyperthermia

Abbreviations: AMF: Alternating Magnetic Field; BSA: Bovine Serum Albumin cm: Centimeter; DH: Hydrodynamic Diameter; DMBA: 7,12-Dimethylbenz[a]anthracene; FBS: Fetal Bovine Serum; g: Gram; HBP: Hamster Buccal Pouch; ICDD: International Centre for Diffraction Data; IgG: Immunoglobulin G; i.m.: Intramuscular; kg: Kilogram; kV: Kilovolt; MEM: Minimum Essential Medium; mg: Milligram; MHT: Magnetohyperthermia; MHz: Megahertz; mL: Milliliter; mm: Millimeter; MNP: (Polyphosphate-coated) Maghemite Nanoparticles; MS: Saturation Magnetization; mV: Millivolt; NIH: National Institute of Health; nm: Nanometer; Oe: Oersted; OSCC: Oral Squamous Cell Carcinoma; PBS: Phosphate Buffer Saline; PCNA: Proliferating Cell Nuclear Antigen; $\mathrm{pH}$ : Potential of Hydrogen; rpm: Rotation Per Minute; TEM: Transmission Electronic Microscopy; U: Units; UM-SCC14A: Squamous Cell Carcinoma of anterior floor of the mouth; UV: Ultraviolet; VSM: Vibrating Sample Magnetometer; XRD: X-Ray Diffraction

\section{Introduction}

Oral squamous cell carcinoma (OSCC), the sixth most common cancer, continues to be the most prevalent cancer related to the consumption of tobacco and alcohol [1], affecting approximately 500,000 patients worldwide each year [2,3]. More than 300,000 new cases are reported in America every year, and approximately 9,000 patients die each year of the disease. Despite several advances in therapeutic regimens, the 5-year survival rate remains approximately $50 \%$ [4]. So, the concept of personalized medicine has been considered the next frontier in the development of solid tumor treatment [5]. Traditionally, oral cancer treatment has been based on the combination of reduction surgery and radiation therapy or chemotherapy to improve the outcome in patients with malignancies in the mouth region. Unlike many other oncology subspecialties, however, the rates of recurrence and overall survival have improved only modestly over the past decades, despite the continuous search for new therapeutic techniques [6,7]. Thus, it is expected that the identification and use of specific agents will lead to individualized treatments, which in turn will lead to significantly better results as manifested by more cures and better quality of life by decreasing toxicity [8].

Over the last years, maghemite nanoparticles (MNPs) have appeared as a well-established technology and an important field of research in superparamagnetism of colloidal materials, characteristic which allows them to be guided with an external magnetic field [9]. Recently, MNPs have been investigated for therapeutic purposes such as hyperthermic treatment [10,11]. Magnetohyperthermia (MHT) represents a novel and promising therapy for cancer treatment $[11,12]$, being capable of promoting the specific lysis of tumor cells, thereby improving patient outcomes while minimizing the subsequent toxicity effects [13]. The

*Corresponding author: Paula Rahal, São Paulo State University "Júlio de Mesquita Filho", UNESP, Institute of Biosciences, Letters and Exact Sciences Department of Biology, Rua Cristovao Colombo, 2265, Jardim Nazareth, CEP 15054-000, São José do Rio Preto, SP, Brazil, Tel: +551732212379; Fax: +551732212390; E-mail: rahalp@yahoo.com.br

Received May 10, 2014; Accepted June 02, 2014; Published June 09, 2014

Citation: Candido NM, Calmon MF, Taboga SR, Bonilha JL, dos Santos MC, et al. (2014) High Efficacy in Hyperthermia-associated with Polyphosphate Magnetic Nanoparticles for Oral Cancer Treatment. J Nanomed Nanotechnol 5: 206. doi:10.4172/2157-7439.1000206

Copyright: ( 2014 Candido NM, et al. This is an open-access article distributed under the terms of the Creative Commons Attribution License, which permits unrestricted use, distribution, and reproduction in any medium, provided the original author and source are credited. 
local heating of biological tissues, which depends on both the applied magnetic field characteristics and the properties of the magnetic sample [14], induces several cellular and pathway changes, including protein denaturation [15]. As a consequence, hyperthermia induces damage to the cytoskeleton, cytoplasm, and organelles membrane, leading to the cell death by apoptosis or necrosis. MHT may be performed using biocompatible nanosized magnetic samples and an appropriate magnetic field-based therapeutic protocol [16]

The hamster buccal pouch (HBP) carcinogenesis model is the bestknown animal system for investigating oral cancer development $[17,18]$ and the therapeutic efficacy of MNPs. The similarities between HBP carcinomas and human OSCCs have provided an expanded rationale to test putative therapeutic agents in the HBP model $[19,20]$. Thus, the present study was designed to evaluate the efficacy of this new potential therapy against oral cancer in vivo that combines MNPs and MHT.

\section{Materials and Methods}

\section{Magnetic nanoparticle preparation and functionalization}

The magnetic nanoparticles used in this work were iron oxide maghemite phase $\left(\gamma-\mathrm{Fe}_{2} \mathrm{O}_{3}\right)$ particles prepared from the oxidation of the precursor phase, magnetite $\left(\mathrm{Fe}_{3} \mathrm{O}_{4}\right)$. Magnetite nanoparticles were synthesized by mixing ferric and ferrous chloride aqueous solutions (2:1 molar ratio) with concentrated ammonia aqueous solution followed by vigorous stirring as described in our previous works [21-23]. The black magnetic precipitate was isolated by magnetic separation, washed with water and oxidized by refluxing in $\mathrm{HCl}$ solution under oxygen flux at $96^{\circ} \mathrm{C}$, yielding a brownish colloidal suspension, indicating the efficient oxidation of magnetite to maghemite. The functionalization of maghemite nanoparticles with tripolyphosphate anions was performed by adding an aqueous sodium tripolyphosphate solution to the acidic maghemite suspension to obtain a tripolyphosphate final concentration of $0.05 \mathrm{~mol} / \mathrm{L}$. The suspension was shaken for 12 $\mathrm{h}$ at room temperature and subsequently dialyzed for $12 \mathrm{~h}$ against demineralized water to eliminate the free tripolyphosphate anions. The $\mathrm{pH}$ of the suspension containing the tripolyphosphate functionalized maghemite nanoparticles was adjusted to 7.0-7.2, and the suspension was purified by centrifugation at $3000 \mathrm{rpm}$ for $5 \mathrm{~min}$ to remove large aggregates. The supernatant was collected and identified as magnetic nanoparticles stock colloidal suspension (MNPs).

\section{Characterizations of the maghemite nanoparticles and of the colloidal suspension}

The $\mathrm{Fe}^{3+/} \mathrm{Fe}^{2+}$ molar ratio in the bare maghemite nanoparticles was obtained using the o-phenanthroline colorimetric method. The total iron concentration of the stock colloidal suspension was determined by atomic absorption spectrophotometry using a Perkin-Elmer 5000 system. X-ray diffraction (XRD) patterns of the bare maghemite nanoparticles were recorded using a XRD-6000 (Shimadzu) X-ray diffraction system. The data were refined using the Basic Process software of the instrument. The cell parameters were calculated using the Unitcell software. The average diameter of the nanocrystalline domain was determined from the full-width at half of the maximum of the 311 reflection peak using the Scherrer's equation [24]. Electronic micrographs of the bare maghemite nanoparticles were obtained with a Jeol 2100 transmission electron microscope. The zeta potential of the functionalized nanoparticles was obtained from electrophoretic mobility measurements performed by phase analysis light scattering using Malvern ZetaSizer Nano ZS equipment. The mean hydrodynamic diameter of the functionalized nanoparticles into the colloidal suspension was also measured using the Malvern instrument. The room temperature nanoparticle magnetization was measured using an ADE vibrating sample magnetometer (VSM) model EV7 with a maximum applied magnetic field of $2 \mathrm{~T}$.

\section{Cell line and MNPs treatment}

Human cancer UM-SCC14A cells from the floor of mouth were kindly provided by Dr. Thomas E. Carey of the University of Michigan, United States. The cells were routinely cultured in Eagle's Minimum Essential Medium (MEM-alpha) (GIBCO BRL, New Zealand) supplemented with $10 \%$ heat-inactivated fetal bovine serum (FBS) and antibiotics $(100 \mathrm{U} / \mathrm{mL}$ penicillin and $0.1 \mathrm{mg} / \mathrm{mL}$ streptomycin $)$ at $37^{\circ} \mathrm{C}$ under $5 \% \mathrm{CO}_{2}$. For the incubation with MNPs, nanoparticles were added to the culture medium at concentrations of $0.35,0.7$ and $1.4 \times 10^{15}$ particle/mL.

\section{MTT assay}

The cell viability assay was performed as previously described [25]. Medium containing MNPs at concentrations of $0.35,0.7$ or $1.4 \times 10^{15}$ particle/mL was added to each well containing $10^{4}$ cells in 96 well plates. After 12 and 24 hours post-incubation with MNPs, the number of metabolically active cells was quantified by measuring the absorbance at $570 \mathrm{~nm}$ in a multiwell plate reader (SpectraMax' Plus 384, USA).

\section{Apoptosis assay}

Medium containing MNPs at concentrations of $0.35,0.7$ and $1.4 \times 10^{15}$ particle $/ \mathrm{mL}$ was added to $2 \times 10^{5}$ cells, and 24 hours postincubation, an apoptosis assay in UM-SCC14A cells was performed as previously described [25].

To analyze the cell death triggered by MNPs, 15 different fields were examined, and the number of apoptotic and unaffected cells were counted to determine the relative rate of death triggered by the different concentrations of MNPs.

\section{Transmission electronic microscopy}

For the qualitative analysis of the cellular uptake efficiency of MNPs by transmission electronic microscopy (TEM), $2 \times 10^{5}$ cells were incubated with $0.7 \times 10^{15}$ particle $/ \mathrm{mL}$ in 6 -well plates for 24 hours. The cells were transferred to a tube to be centrifuged at $1000 \mathrm{rpm}, 4^{\circ} \mathrm{C}$ for 20 minutes. The supernatant was removed, and the cells were washed twice with PBS solution.

After the supernatant was removed and the cells were washed PBS solution, the cells were fixed for two hours in $4 \%$ paraformaldehyde and $0.5 \%$ glutaraldehyde at $4^{\circ} \mathrm{C}$, followed by a $10 \%$ fixing solution and $90 \%$ sodium cacodylate buffer ( $\mathrm{pH}$ 7.4). The samples were rinsed with sodium cacodylate buffer, and dehydration was performed in stepwise increasing concentrations of methanol. Finally, the samples were embedded in a pre-mixture of equal parts of LR gold (London ResinCo., Reading, Berkshire, UK) and 100\% methanol for 24 hours at $-20^{\circ} \mathrm{C}$. The contents of the capsules were centrifuged at $1500 \mathrm{rpm}$ at $4^{\circ} \mathrm{C}$ for 20 minutes and the polymerization was performed out using pure LR gold. Finally, LR gold and $0.25 \%$ activator solution were added to the capsules, followed by exposure to UV light for approximately 24 hours at $-20^{\circ} \mathrm{C}[26]$.

The embedded cells were cut using a microtome (Ultracut UCT, Leica, Germany) into $50 \mathrm{~nm}$ ultrathin sections using a diamond knife, placed on copper grids and then post-stained with uranyl acetate and lead citrate. Transmission electron microscopy was performed using a Leo-Zeiss 906 EXII at $80 \mathrm{kV}$, and the images captured with a CCD 
camera (Veleta, Germany). Cells that were not incubated with MNPs were used as controls.

\section{Animals}

Thirty male 8-week Syrian hamsters (Mesocricetus auratus) were purchased from Anilab Laboratory Animals. Animals were maintained on a standard laboratory diet, and feed (purchased from commercial supplier) and water were provided ad libitum. The animals were housed at a density of five animals per cage and were kept at room temperature $\left(22 \pm 2^{\circ} \mathrm{C}\right)$ with $50 \pm 10 \%$ humidity and an automatically controlled cycle of 12 hours light and 12 hours dark. All animal experiments were performed in accordance with the NIH "Guide for the Care and Use of Laboratory Animals", and experimental protocols were approved by the Committee of Ethics in Animal Research, São Paulo State University, SP, Brazil (Protocol number: 029/2010)

\section{Experimental design}

After acclimation for one week, the hamsters were randomly divided into five groups $(n=6)$. With the exception of animals in the control group that served as a negative control (Normal group), all hamsters were used as animal models of oral cancer, and a portion of them were treated with magnetohyperthermia and MNPs treatment. The right buccal pouches of these hamsters was topically treated with 0.5\% DMBA in mineral oil (7,12-dimethylbenz[a]anthracene, Sigma Chemical, St Louis, MO) with a paintbrush three times/week for 16 weeks.

Tumor sizes typically reached $200-250 \mathrm{~mm}^{3}$, and one group was selected to be the tumor control (Cancer group), in which no treatment was performed. Two days after the last DMBA using, the hamsters in the MNP group received injections of the nanoparticles suspended in the magnetic fluid $\left(0.7 \times 10^{15}\right.$ particle $\left./ \mathrm{mL}\right)$. This procedure was performed under anesthesia with ketamine [dopalen, Vetbrands, Brazil, $60 \mathrm{mg}$ applied per $\mathrm{kg}$ bodyweight, intramuscular (i.m.)] and xylasine (anasedan, Vetbrands, Brazil, $10 \mathrm{mg} / \mathrm{kg}$, i.m.).

Additionally, two groups were separated to combine nanoparticle therapy with animal exposure to an alternating magnetic field (AMF) generated by equipment operating at the frequency of $1 \mathrm{MHz}$ and 40 Oe field amplitude [16]. The procedure for the magnetic field exposure was performed over 15 minutes for three consecutive days after the MNP application. One group was euthanized one day post-treatment (MNP+AMF1 group), and another group was euthanized after seven days (MNP+AMF7 group) to check the response to therapy over different periods of animals monitoring.

Hamsters were euthanized and exsanguinated. Blood samples were collected to analyses of white cell counts, and all animals were weighed. After necropsy, the organs were inspected macroscopically. Tissue samples of the buccal pouches were collected for histopathological and immunohistochemical examination. Buccal pouches (right and left), livers, hearts, lungs, kidneys and spleens were collected for organ evaluation and verification of adverse reactions to the MNPs or treatment.

\section{Histopathological analysis}

The histopathological analysis was performed as previously described [27].

\section{Immunohistochemical staining}

The immunocytochemistry staining was performed as previously described [27] with modifications (see Supplementary Material). The primary antibody anti-PCNA (sc56, PC10 clone, IgG mouse monoclonal, Santa Cruz Biotechnology, USA) was diluted 1:100 in 1\% bovine serum albumin (BSA), and the primary antibody anti-caspase
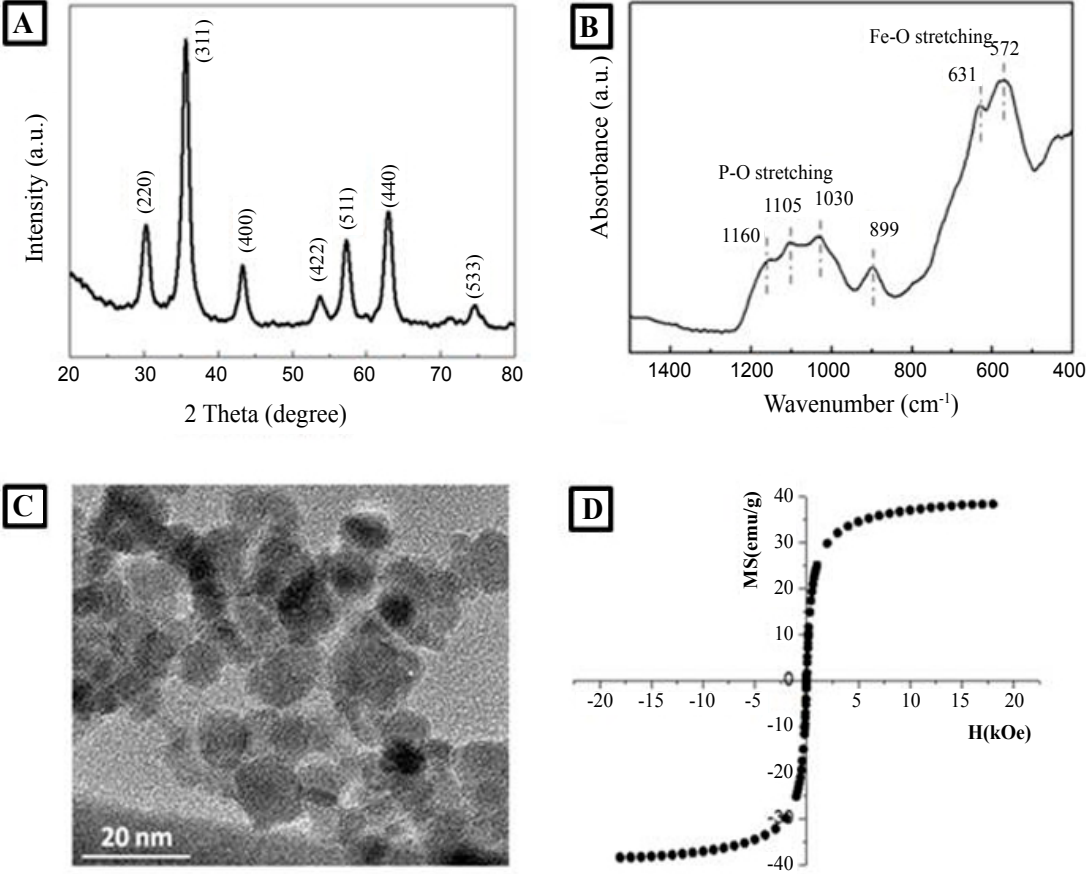

Figure 1: A. X-ray diffractogram of bare MNPs. B. Infrared spectrum of tripolyphosphate functionalized MNPs. C. Transmission electron micrograph of functionalized MNPs. D. Magnetization versus magnetic field plot of the functionalized MNPs. 
3 (ab13847, IgG rabbit polyclonal, Abcam, USA) was diluted 1:50 in $1 \%$ BSA.

\section{Statistical analysis}

All experiments were performed in triplicate in two independent assays, and the data are expressed as the mean \pm standard deviation. The standard deviation values are indicated as error bars in the subsequent graphs. The results were statistically processed for outlier detection using the GraphPad Prism 5 software (GraphPad Inc., San Diego, CA, USA). Statistical judgments were determined by analysis of variance ("one-way" ANOVA) followed by Tukey's post-test, during which results were considered statistically significant at the $95 \%$ confidence interval (i.e., $\mathrm{p}<0.05$ ).

\section{Results}

\section{Characterization of the maghemite nanoparticles and colloidal suspension}

The chemical analysis of the bare nanoparticles revealed a $\mathrm{Fe}^{2+} \mathrm{Fe}^{3+}$ molar ratio of 0.02 , demonstrating an efficient oxidation process. The total iron content in the stock colloidal suspension was $17.5 \mathrm{mg} / \mathrm{mL}$. The XRD diffractogram (Figure 1A) demonstrated a pattern characteristic of the cubic spinel $(F d 3 m)$ structure. The lattice unit cell parameter calculated from the diffractogram was $8.347 \AA$, which is closer to the value reported in the ICDD (PDF No. 39-1346.) for bulk maghemite $(8.351 \AA)$. These chemical compositional and crystallographic data characterize the magnetic nanoparticles as iron oxide maghemite phase. Moreover, in the infrared spectrum of the tripolyphosphate functionalized nanoparticles shown in Figure 1B, the bands observed at 631 and $572 \mathrm{~cm}^{-1}$ are related to the vibrational stretching

modes characteristic of the Fe-O bonding in the tetrahedral and octahedral sites of maghemite, respectively [28]. The set of bands between 850 and $1200 \mathrm{~cm}^{-1}$ are attributed to P-O stretching of bonds encountered in the tripolyphosphate molecules bonded to the nanoparticles surface [29].

Transmission electron microscopy analysis revealed nanoparticles of irregular morphology and broad size distribution (Figure 1C). The values of the average particle diameter and standard diameter deviation obtained by fitting the nanoparticles counting data were $8.12 \pm 0.14$ $\mathrm{nm}$ and $0.35 \pm 0.01$, respectively. The hydrodynamic diameter $(\mathrm{DH})$ of the nanoparticles into the colloidal suspension was $80 \mathrm{~nm}$ in the $\mathrm{pH}$

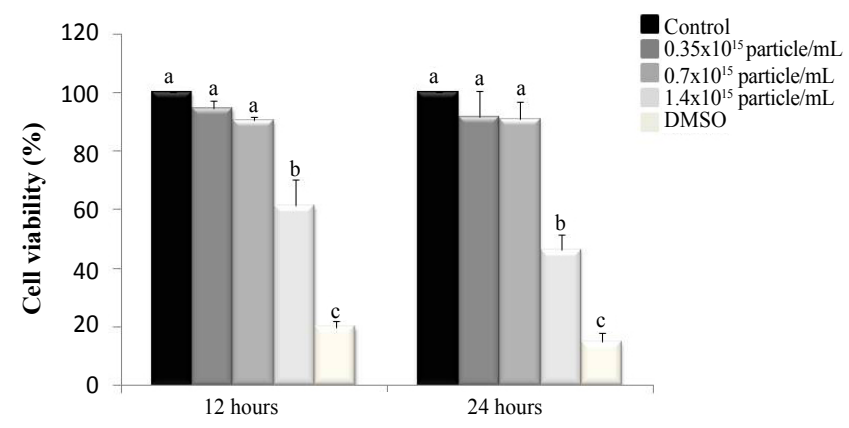

Incubation time

Figure 2: Effect of MNPs on cell proliferation. MTT assay of UM-SCC14A cells after 12 and 24 hours of incubation with MNPs. Data are the mean \pm SD. Within graphs, columns with different superscript letters differ significantly $(p<0.01)$
7.2. Because the mean diameter of the nanoparticles as determined by TEM was approximately $8 \mathrm{~nm}$, the value of $\mathrm{DH}$ obtained indicate that the nanoparticles exist as very small aggregates into the colloidal suspension. The zeta potential of these small nanoparticles aggregates into the colloidal suspension at $\mathrm{pH} 7.2$ was $-43.4 \mathrm{mV}( \pm 3.55)$. As the zeta potential of bare maghemite particles at $\mathrm{pH} 7$ is approximately zero [30], the value obtained is also evidence of the effective functionalization of the nanoparticles with tripolyphosphate, from which the PO3- groups provide a high negative charge density on the nanoparticles surface. In addition, the higher surface charge density on the nanoparticles aggregate prevents its secondary aggregation, yielding a suspension with high colloidal stability.

The magnetic property of the tripolyphosphate functionalized maghemite nanoparticles was measured by a vibrating sample magnetometer (VSM). Figure 1D presents the magnetization as a function of an external field at ambient temperature. The curve presents no hysteresis (zero coercivity), indicating that the nanoparticles exhibited superparamagnetism at room temperature. The observed saturation magnetization (MS) obtained was $39 \mathrm{emu} / \mathrm{g}$. This value is smaller than the values reported for bulk maghemite at $300 \mathrm{~K}$ (MS=76 emu/g) [31] but is similar to the MS values previously reported for maghemite nanoparticles in the range size of $5-10 \mathrm{~nm}$ [32]. The difference in the saturation magnetization between the bulk and the nanosized material can be attributed to the small particle size effect [33].

\section{Effect of MNPs on proliferation in oral cancer cell line}

The cells retained their viability for both 12 and 24 hours in the experimental control (without MNP incubation). Moreover, the number of viable cells was time-dependent and MNP dose-dependent. Thus, in cells incubated with different MNPs concentrations, we observed a decrease in the average absorbance and attenuation in cell viability (Figure 2).

No significant difference in cytotoxicity was observed for MNPs up to $0.7 \times 10^{15}$ particle $/ \mathrm{mL}$, irrespective of the incubation time with MNPs (cell viability approximately 90\%), indicating nontoxic nature at these concentrations. However, at the $1.4 \times 10^{15}$ particle $/ \mathrm{mL}$ concentration, the cytotoxicity increased. This increase was verified by the significant cell viability decrease over 12 and 24 hours of MNP exposure $(\mathrm{p}<0.01)$ (Figure 2). Then, UM-SCC14A cell treatments with different concentrations of these MNPs resulted in cell proliferation suppression in a dose- and time-dependent manner.

\section{Apoptosis assay}

Although MNPs were not administered to control cells, apoptosis was also observed in this case because it is a common physiological process (Figure $3 \mathrm{~A}-\mathrm{C}$ ). In addition to exhibiting cell growth similar to the control, the cell line incubated with 0.35 and $0.7 \times 10^{15}$ particle/ $\mathrm{mL}$ also exhibited typical signs of programmed cell death (Figure 3 $\mathrm{D}-\mathrm{G}$ and $\mathrm{H}-\mathrm{K}$, respectively). After exposure to the toxic concentration $\left(1.4 \times 10^{15}\right.$ particle $\left./ \mathrm{mL}\right)$, it was possible to observe signs of damage (Figure 3L-O). Most cells have died at this concentration, and even the surviving cells have sustained serious damage. This damage can likely be attributed to the increased cytotoxicity of the cellular environment as well as the large number of MNPs when cells were incubated with $1.4 \times 10^{15}$ particle $/ \mathrm{mL}$.

Finally, we observed in a quantitative manner that the level of programmed cell death gradually increased in a dose-dependent manner (Figure 3P). The difference between samples was highly 
Control

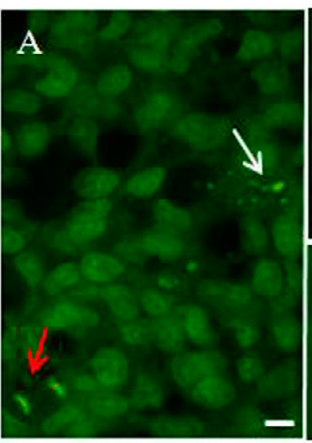

$0.7 \times 10^{15}$ particle $/ \mathrm{mL}$
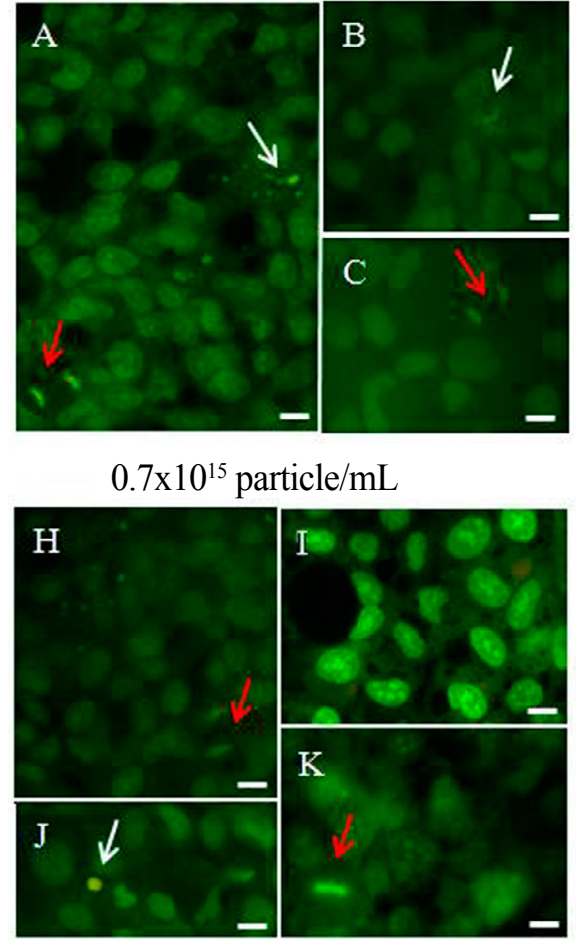

$0.35 \times 10^{15}$ particle $/ \mathrm{mL}$
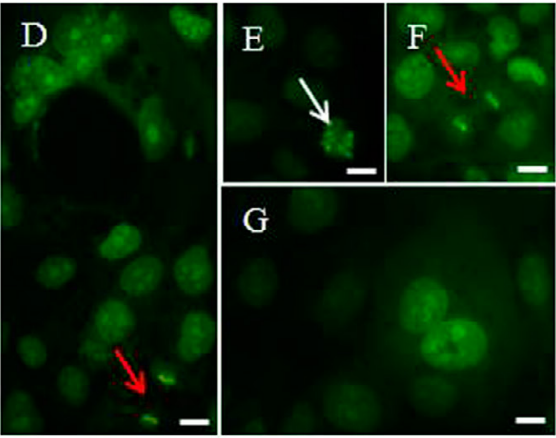

$1.4 \times 10^{15}$ particle $/ \mathrm{mL}$
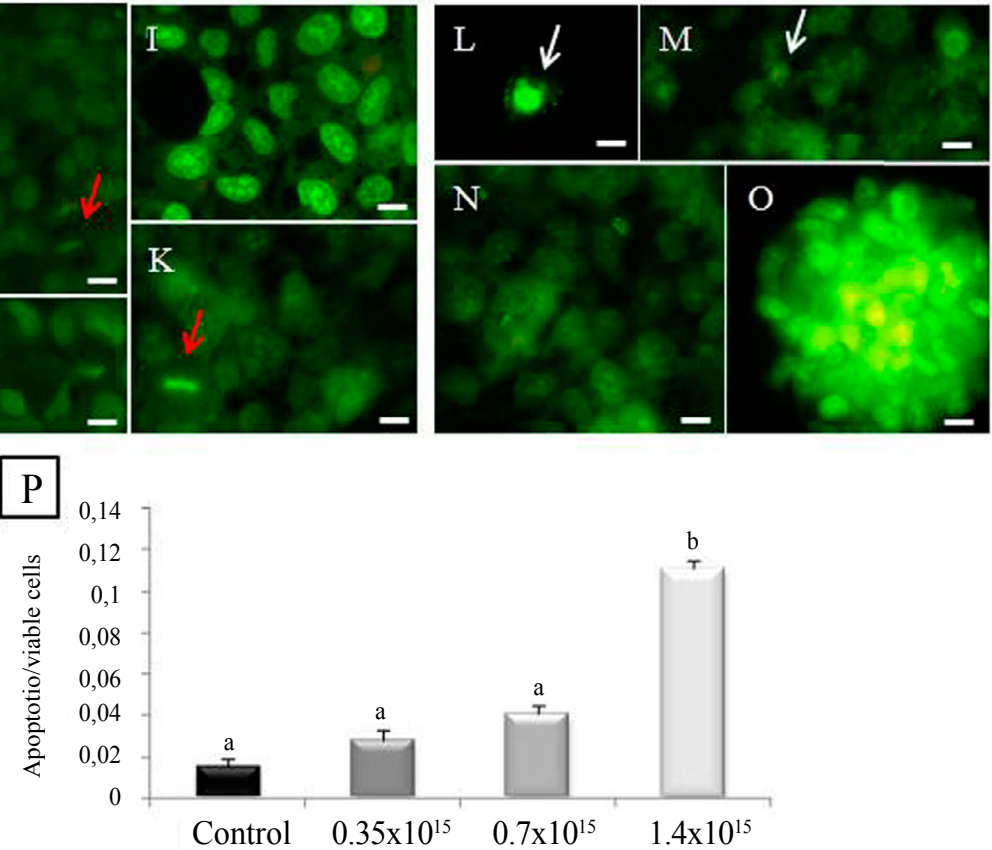

Figure 3: Effect of MNPs on apoptosis. Acridine orange and alumen ferric staining of UM-SCC14A cells analyzed 24 hours post-incubation with or without MNPs. (A-C) Control experiment without MNP incubation. (D-G) Cells incubated with $0.35 \times 10^{15},(\mathrm{H}-\mathrm{K}) 0.7 \times 10^{15}$ and $(\mathrm{L}-\mathrm{O}) 1.4 \times 10^{15}$ particle/mL. Scale bars represent 10 $\mu \mathrm{m}$. White arrows indicate cells in apoptosis, and red arrows indicate cells in the process of mitosis. (P) Graphic of the rate of apoptosis. Data are the mean \pm SD. Within graphs, columns with different superscript letters differ significantly $(p<0.001)$.

significant only with respect to the largest concentration of MNPs when compared with other samples $(p<0.001)$, demonstrating its toxic character for this cell type.

\section{Ultrastructural analysis of UM-SCC14A cells and in vitro cell uptake}

Due to the mitotic activity of the cell line, it was possible to identify cells that exhibited an extensive complement of cytoplasmatic organelles, especially mitochondria and vacuoles. In many cells, fingerlike projections in plasma membrane, including microvilli, were observed. In addition to this abundance of organelles, the neoplastic nature of these cells was suggested by the large number of nuclei and nucleoli (Figure 4A-C). Overall, there were no differences between the experimental groups with respect to cell ultrastructure.

The abundance of cytoplasm and mitochondria and the richness of surface microvilli exhibited by the cells demonstrated the intense metabolic activity of these cancer cells, confirming the non-toxic feature of these MNPs at concentration of $0.7 \times 10^{15}$ particle $/ \mathrm{mL}$. Comparing the appearance of the cells with and without MNPs incubation, we observed that cells exposed to MNPs exhibited an elevated number of vacuoles and vesicles. Magnification of the interior of the vacuoles indicated the presence of electron-opaque regions, which represent agglomerates of particles, thereby confirming the efficient internalization of biocompatible polyphosphate-coated MNPs in this cell type (Figure $4 \mathrm{D}-\mathrm{F})$. Thus, the particles were sequestered into these vacuoles after penetration and were destined for degradation. Such organelles were eletrolucid (autophagic vacuoles) or eletrodense (endosomes). This confirmed that UM-SCC14A cells were able to internalize these MNPs and that the procedure was successful, demonstrating no extreme ultrastructural differences between the cells incubated with the MNPs when compared with the control. 

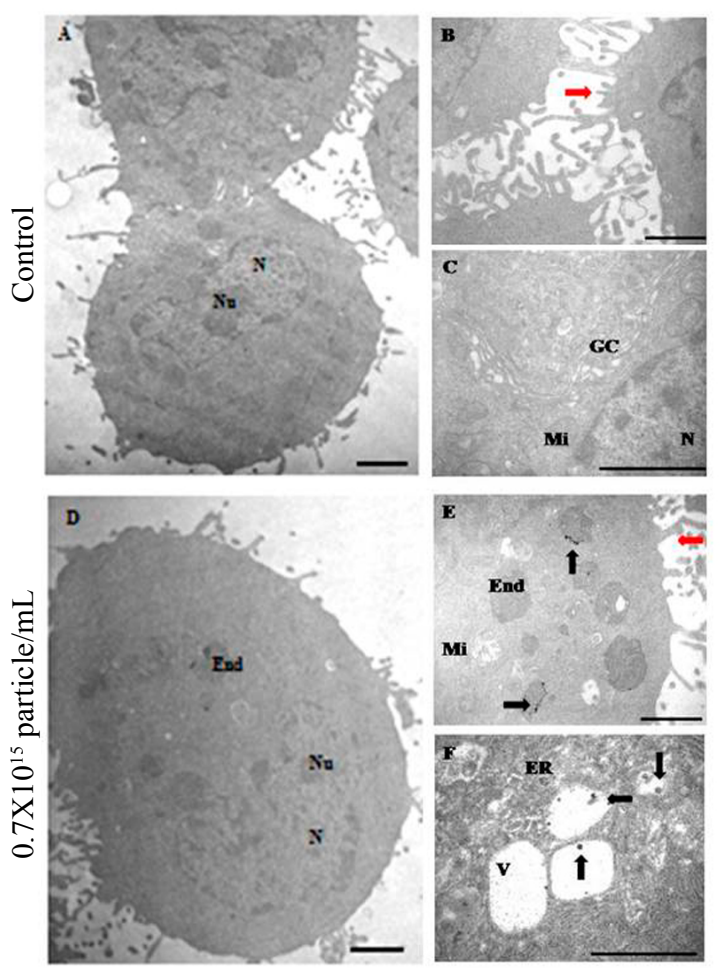

Figure 4: TEM images of the UM-SCC14A cell line. (A-C) Control cells without MNP-incubation and (D-F) cells incubated with $0.7 \times 1015$ particle/ $\mathrm{mL}$ for 24 hours. Scale bars represent $500 \mathrm{~nm}$. Black arrows indicate MNPs internalized by eletrolucid vesicles or eletrodense vesicles in the cytoplasm. Red arrows indicate projections of the plasma membrane. GC: Golgi complex. End: eletrodense endosome. ER: endoplasmatic reticulum. Mi: mitochondria. N: nucleus. Nu: nucleolus. V: eletrolucid autophagic vacuole.

\begin{tabular}{|c|c|c|c|c|}
\hline \multirow{2}{*}{ Experimental group } & \multicolumn{3}{|c|}{ Mucosa } & \multirow{2}{*}{ Total } \\
\cline { 2 - 4 } & Normal & Hyperkeratosis & OSCC & \\
\hline Normal & 6 & 0 & 0 & 6 \\
\hline Cancer & 0 & 6 & 6 & 6 \\
\hline MNP & 2 & 5 & 4 & 6 \\
\hline MNP+AMF1 & 6 & 3 & 0 & 6 \\
\hline MNP+AMF7 & 6 & 0 & 0 & 6 \\
\hline
\end{tabular}

OSCC: oral squamous cell carcinoma. MNP: polyphosphate-coated magnetic nanoparticles. AMF1 and AMF7: data collected one day and seven days respectively, after magnetohyperthermia procedure.

Table 1: Effect of MNPs and MHT treatment on DMBA-induced oral carcinogenesis in hamsters.

\section{Clinical observations}

No incidents of mortality as a consequence of MNP administration and/or AMF were observed in any of the treated groups. There were no signs of general clinical symptoms of toxicity or abnormal behavioral reactions.

\section{Tumor histopathology}

In the normal group (without oral tumor induction), normal cell and tissue characteristics were visualized (Table 1), including stratified squamous epithelium and a keratin layer with regular thickness (Figure $5 \mathrm{~A}$ ). In the cancer group (oral tumor induced tumor by the DMBA carcinogen), some general features that distinguish malignant cells from normal cells were observed, characterizing OSCC.

In the Cancer group (Figure $5 \mathrm{~B}$ ), it was possible to observe cellular pleomorphism and a large number of atypical mitoses. With respect to tissue arrangement, hyperplasia, hypergranulosis, hyperkeratosis, parakeratosis and acanthosis was observed in the basal layer. Papillomatosis was also observed to a lesser degree, and in the affected area, a mixed inflammatory infiltrate and neovascularization were noted. In addition, there was an irregular extension of the epithelial lesion through the basal membrane into the conjunctive tissue as well as invasive epithelial squamous cells within the tissue that were not connected to the epithelium. Similarly, keratin pearls were present in focal round areas from keratinized cells (Table 1).

The features described above were also observed, to a slightly lesser extent, in animals treated with MNPs (Figure 5C). There was a slight recovery in the tumor size of group MNP but without complete regression (Figure 5C and Table 1). However, in animals that received nanoparticles treatment in conjunction with exposure to a hyperthermia-inducing alternating frequency magnetic field (MNP+AMF1 and MNP+AMF7 groups), we observed a significant regression of the cancerous state. Furthermore, the response to therapy was time-dependent, as the results were more satisfactory for the animals euthanized after seven days (Figure 5E) compared with the animals euthanized one day after treatment (Figure 5D). This favorable response was validated by observing that the tissue nearly normal despite the large number of the cells that underwent apoptosis and that it lacked the features described in the group of animals with carcinoma in situ.

In quantitative terms of the magnetotherapy efficacy involving these polyphosphate-coated MNPs, $100 \%$ recovery (12/12) was observed in the Syrian hamsters treated by this manner (Table 1). This fact supports the tumor regression observed in hamsters treated by these MNPs with AMF.

\section{Biometric analysis}

The biometric analysis revealed no significant difference in the bodyweight of animals in Normal and Cancer groups. Animals treated with the MNPs likely experienced slight discomfort on the first day after treatment because the region is crucial for feeding. This fact was verified due to the statistically significant difference $(\mathrm{p}<0.05)$ in the body weight between MNP+AMF1 animals and the control groups.

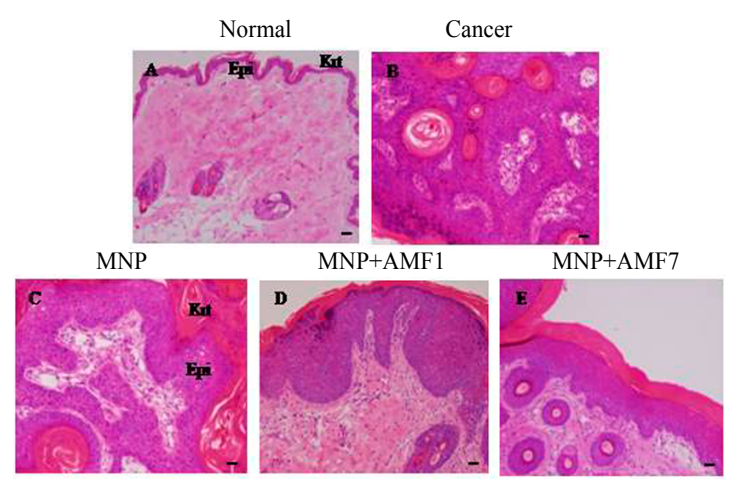

Figure 5: Histopathological sample of the right buccal pouch in the $(A)$ Normal, (B) Cancer, (C) MNP, (D) MNP+AMF1 and (E) MNP+AMF7 groups. Tissues were stained by HE. Scale bars represent $20 \mu \mathrm{m}$. Epi: epithelium. Krt: keratin. 

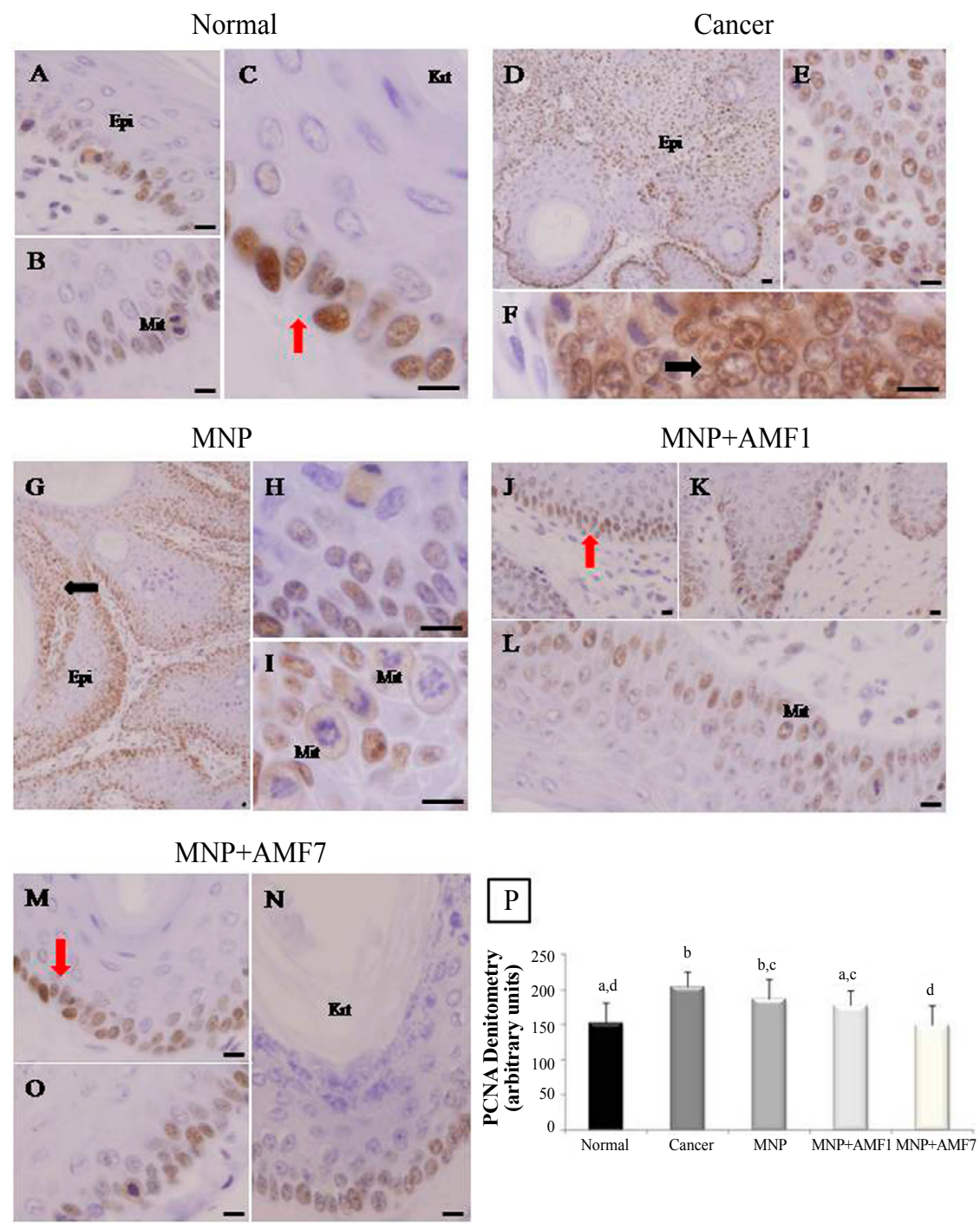

Figure 6: Histological sections subjected to immunocytochemistry for the detection of the cell proliferation marker PCNA. Slides were counterstained with Harris hematoxylin. PCNA immunostaining of epithelial cells in buccal pouch tissue of male hamsters in the (A-C) Normal, (D-F) Cancer, (G-I) MNP, (J-L) MNP+AMF1 and (M-O) MNP+AMF7 groups. Red arrow: marking limited to the basement membrane of epithelium. Black arrow: atypical mitosis and dispersed marking throughout the tissue, with increased PCNA expression. Scale bars represent $10 \mu \mathrm{m}$. Epi: epithelium. Krt: keratin layer. Mit: mitosis. (P) Densitometric analysis. Data are the mean $\pm S D$. Within graphs, columns with different superscript letters differ significantly $(p<0.05)$.

However, the animals evidently adapted near-normal body mass seven days after the magnetohyperthermia therapy (Figure S1 A).

\section{Morphometric analysis}

In the DMBA cancer-induced groups, we observed a compromised corrugated and deformed configuration that disturbed the tissue structure pattern, exhibiting lesions and epithelial projections into the conjunctive tissue. This led to major restructuring in tissue when compared with the Normal group (Figure S1 B and C).

The general morphology of the oral tissue exhibited a significantly smaller epithelium layer in the Normal group compared with the Cancer and MNP groups $(\mathrm{p}<0.001)$. However, there was a major reduction in the structural thickness of the epithelium of the animals exposed to magnetohyperthermia treatment $(\mathrm{MNP}+\mathrm{AMF} 7)$ compared with the neoplastic group $(\mathrm{p}<0.001)$. There was a clear recovery of these morphological characteristics, resembling the epithelium of normal animals (Figure S1 B).

The values obtained for the control group (Normal) were similar to MNP+AMF1 and MNP+AMF7 ( $>0.05)$ with respect to the morphometric analysis of the keratin layer thickness (Figure S1 C), demonstrating the major restructuring in the arrangement of the buccal pouch tissue. However, for the Cancer and MNP groups, the corneum stratum thickness significantly increased $(p<0.001)$ when compared with the Normal group, confirming the presence of the tumor and confirming that treatment with MNPs alone is insufficient for a positive cancer response.

The tissues of hamsters treated with hyperthermia exhibited a reversal in the cell progression and carcinogenic process as evidenced by cell morphology, which was characterized by reductions in the 
epithelial cell height and keratin layer thickness. These results revealed that the tumor regressed in the experimental groups treated with magnetohyperthermia, with a small variation in the data set.

\section{Immunohistochemical analyses of PCNA and active caspase-3}

We observed extremely low PCNA immunostaining, which was located only in the basal layer of the stratified squamous epithelium present in the mucosa in normal animals (Figure 6A-C) and in animals treated with magnetohyperthermia (Figure 6J-O) when compared with animals with oral cancer (Figure 6D-F) and those treated with MNPs (Figure 6G-I), which exhibited a pronounced increase and diffuse expression of PCNA throughout the tissue.

A significant increase in the PCNA endogenous protein expression (Figure 6P) was observed in the Cancer and MNP groups, representing increased and uncontrolled cell proliferation. Furthermore, the expression of PCNA did not differ among Normal, MNP+AMF1 and MNP+AMF7 oral tissues ( $p>0.05$ ). In contrast, there was a significant difference $(\mathrm{p}<0.05)$ between the Cancer group compared with the $\mathrm{MNP}+\mathrm{AMF1}$ and MNP+AMF7 groups (Figure 6P).

Extensive active caspase- 3 staining was localized largely in the upper layer of the epithelium near the keratin layer in normal tissues (Figure 7A-C) and in animals treated with magnetohyperthermia (Figure $7 \mathrm{H}-$ L). However, reduced staining was observed in animals with OSCC (Figure 7D-E) and in animals treated only with MNPs (Figure 7F-G).

The quantitative analysis by densitometry (Figure $7 \mathrm{M}$ ) demonstrating elevated caspase-3 labeling in the Normal, MNP+AMF1 and MNP+AMF7 groups. No significant difference in caspase-3 labeling was observed between the Cancer and MNP groups ( $\mathrm{p}>0.05)$,
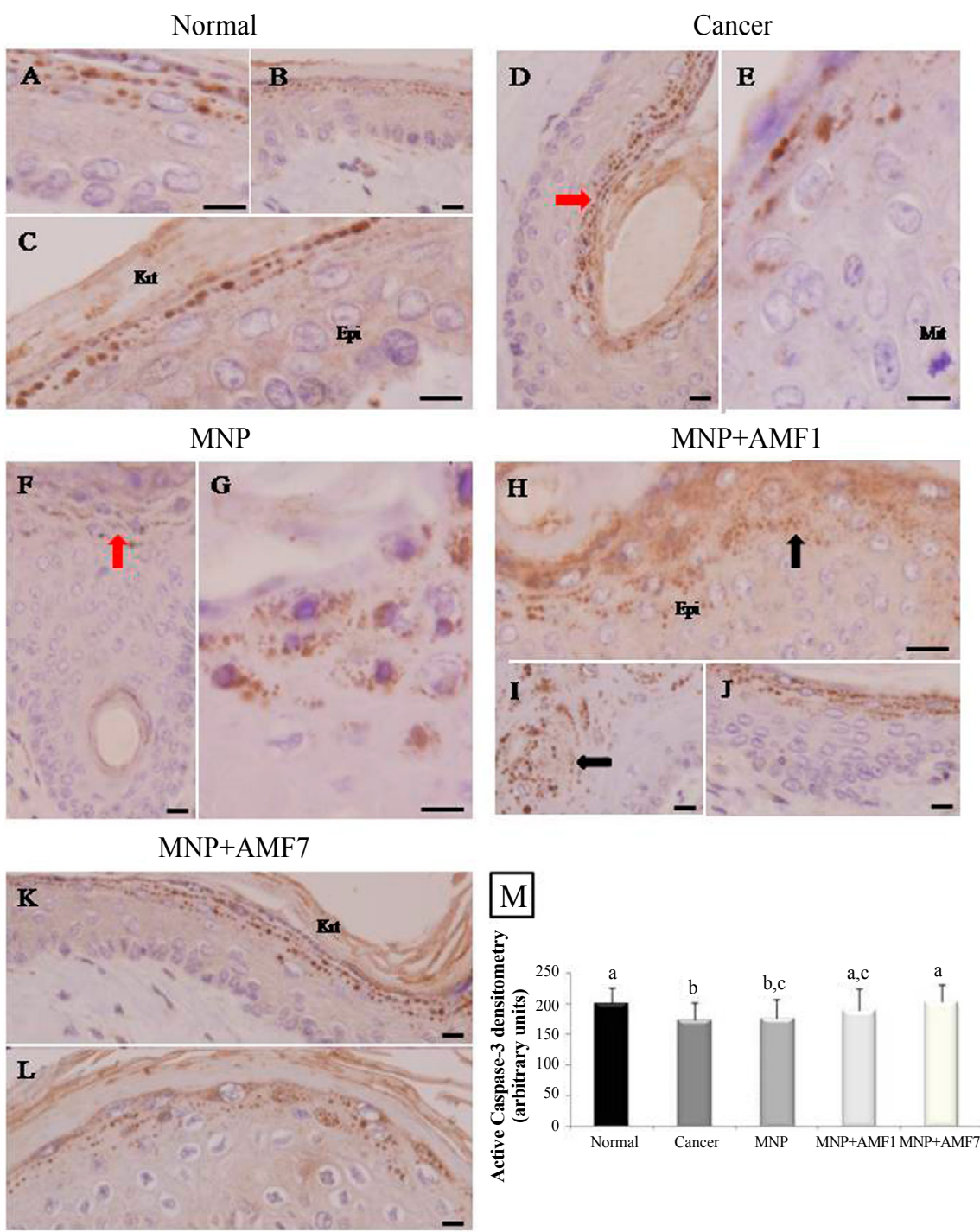

Figure 7: Immunocytochemical analysis of active caspase-3, counterstained with Harris hematoxylin. Active caspase-3 immunostaining of epithelial cells in buccal pouch tissue of male hamsters in the (A-C) Normal, (D-E) Cancer, (F-G) MNP, (H-J) MNP+AMF1 and (K-L) MNP+AMF7 groups. Cancer and MNP groups exhibit immunostaining limited to the top layer of the epithelium or the islands of keratin (red arrows), whereas the Normal, MNP+AMF1 and MNP+AMF7 groups exhibited, in addition to markings in the aforementioned region of the epithelium, significant markings dispersed in lower layers (black arrows). Scale bars represent $10 \mu \mathrm{m}$. Epi: epithelium. Krt: keratin layer. Mit: mitosis. (M) Densitometric analysis. Data are the mean \pm SD. Within graphs, columns with different superscript letters differ significantly $(p<0.05)$ 
but a significant difference was observed $(\mathrm{p}<0.001)$ when the Cancer and MNP groups were compared with the Normal group.

\section{The proinflammatory effects of the MNP administration}

The total leukocyte count indicated variations among the different experimental groups, and the results obtained on the sacrifice days are presented in Figure S2. In hamsters with oral carcinoma, we observed an increased inflammatory response, which was confirmed by the significant difference $(\mathrm{p}<0.05)$ with respect to the Normal group.

However, in animals treated with magnetohyperthermia, there was a significant decrease in the leukocyte population compared with the Cancer group $(\mathrm{p}<0.05)$. Thus, a recovery of the normal white cell count was observed, with no statistically significant differences between the Normal and MNP+AMF groups ( $p>0.05)$. Furthermore, although not as obvious, the animals treated with MNP also exhibited no significant difference $(\mathrm{p}>0.05)$ in the leukocyte population when compared with the normal group and with the tumor bearing animals (Figure S2).

\section{Internal organs and MNPs biodistribution}

In general, throughout the period of observation, there were no clinically important effects, and all fluctuations of the tissue parameters remained within the physiological range. Thus, there was no change observed in the histopathology of the removed tissues (left buccal pouches, livers, hearts, lungs, kidneys and spleens) of the animals from the groups analyzed (data not shown).

Intracellular MNPs were not observed in most organs. Nevertheless, MNPs were observed at exceptionally high levels in the spleen. A variable amount of small blue clusters was observed in 83\% (5/6) of the animals' spleens in the MNP+AMF1 group. This observation demonstrated that the biodistribution of such nanoparticles synthesized with iron oxide and polyphosphate coated somehow passed through the spleen before being removed from the body (Figure S3 A-E). However, we also demonstrated that there was no accumulation of MNPs in the body; after a few days of treatment, these nanoparticles were not found anymore.

\section{Discussion}

Magnetic nanoparticles have been successfully used as a potential anticancer drug carrier or as an antenna to relay externally applied energy for hyperthermia therapeutic purposes [34]. Combined with hyperthermia, nanotechnology exhibits a wide range of application possibilities in cancer therapy $[35,36]$, in particular for the treatment of oral carcinoma. The biokinetics of nanomaterials depends on their size, shape, chemical composition, surface structure, solubility and aggregation capacity. These parameters can modify the cellular uptake, the accumulation at the target site in vivo and the possibility of causing tissue damage [37,38].

Some experiments suggest that nanoparticles with diameters in the range of 8 to $15 \mathrm{~nm}$ are less toxic than those smaller than $8 \mathrm{~nm}$ or larger than $15 \mathrm{~nm}$ [39]. The results of our particle size analysis indicated that the mean size of the particles used in our system is in this adequate nanometric range (average diameter of $8 \mathrm{~nm}$ ). The surface charge or zeta potential $(\zeta)$ is the electrokinetic potential formed by an ionic double layer that is susceptive to a voltage difference in millivolts [40]. The measurement of $\zeta$ allows predictions of the stability of colloidal aqueous dispersions. Typically, aggregation is less likely to occur in particles with a high positive or negative zeta potential due to electric repulsion [41], as observed in our study $(-43.4 \mathrm{mV})$. Therefore, because of their ultra-fine size, high biocompatibility and magnetic characteristics, the nanoscale compound in our study exhibited suitable features for an intermediate for magnetohyperthermia.

As corroborated by TEM, MNPs were efficiently taken up by UMSCC14A cells through endocytosis and were trapped in endosomes due to their favorable nanoscale characteristics. These endosomes then fused with lysosomes for processing before being transported to the cell periphery for excretion [42]. In cell cultures, high doses of iron oxide MNPs can be harmful, altering the cell growth rate [43]. As demonstrated by the apoptosis assay, salient morphological changes were observed, including cell rounding, membrane blistering and nucleus fragmentation into apoptotic bodies, all characteristics of apoptotic cells $[44,45]$. Moreover, when exposed to MNPs, the number of viable cells decreased with time and with increasing concentrations of MNPs, thereby demonstrating significant attenuations in cell viability when the cells were incubated with concentrations higher than $0.7 \times 10^{15}$ particle $/ \mathrm{mL}$.

We observed that the magnetohyperthermia treatment exhibited two notable effects. First, treatment resulted in intense apoptosis and fibrosis in the tumor area, as evidenced by the increase in the expression of active caspase-3. Second, treatment inhibited cell proliferation, which was confirmed by decreased PCNA immunostaining. These results demonstrate that the antitumor effectiveness of magnetohyperthermia treatment can be associated with cell death in the target tissue and is consistent with the tumor regression observed in hamsters treated with this magnetic fluid composition and AMF. The treatment of tumors via magnetic heating has already demonstrated promising results in other tumors [16,46-49].

Another concern of the use of MNPs in biomedical applications is the elimination of this nanomaterial [50]. There are different mechanisms in vivo that govern the behavior of MNPs, and it has been noted that different shapes and surface charges of the particles influence their biological distribution [16]. The fact that in our experiment, only the spleen was selective for this type of MNP can be explained by its status as a secondary lymphoid organ that degrades erythrocytes. This fact suggests that the clusters observed in the spleen were MNPs captured by mononuclear phagocytes that partially or totally lost coverage and, without steric repulsion between them, lost stability and agglomerated [51]. Therefore, the spleen was the organ associated with the biokinetics and elimination of MNPs. In this work, we observed no severe changes in the presence of $0.7 \times 10^{15}$ particle $/ \mathrm{mL}$; these organic compounds, functionalized iron oxide MNPs, exhibited not only the basic magnetism characteristics of magnetic nanoparticles but also exhibited the biocompatibility and biodegradability of functional organic materials [52].

The last variable investigated in this work was the inflammatory response to the treatment. The leukocyte count is an important tool for pathological analysis because it can indicate inflammatory and allergic processes [53]. This response is of great importance in the evaluation of new nanobiotechnology materials, including magnetic fluids and magnetoliposomes $[53,54]$. In this study, the MNP sample exhibited proinflammatory potential without cause, however, no significant histopathological changes, consistent with the results of Portilho et al. [16]. Thus, these results demonstrate the biocompatibility of MNPs and are of great interest in the field of nanomedicine as an effective treatment of OSCC.

We suggest the use of a concentration of $0.7 \times 10^{15}$ particle $/ \mathrm{mL}$ for 
Citation: Candido NM, Calmon MF, Taboga SR, Bonilha JL, dos Santos MC, et al. (2014) High Efficacy in Hyperthermia-associated with Polyphosphate Magnetic Nanoparticles for Oral Cancer Treatment. J Nanomed Nanotechnol 5: 206. doi:10.4172/2157-7439.1000206

therapeutic application free of adverse effects because this concentration did not cause significant cytotoxicity in vitro. Furthermore, in this study, we reported the successful application of biocompatible MNPs with magnetohyperthermia and the subsequent in vivo lysis of oral squamous cell carcinoma. This antitumor effect of the magnetohyperthermia treatment was followed by the inhibition of cell proliferation and by tumor tissue injury characterized by apoptosis and fibrosis. Remarkably, this report is the first to demonstrate the therapeutic efficacy of these MNPs in oral cancer. This fact emphasizes that further investigation into the MHT process is required to improve patient outcomes. Our findings suggest this MNP as a potential nanoparticle to be applied in the MHT treatment of patients with OSCC.

\section{Acknowledgement}

L. R. Falleiros Jr.'s technical assistance is gratefully acknowledged (from São Paulo State University "Júlio de Mesquita Filho", UNESP, Institute of Bioscience, Language \& Literature and Exact Science, Department of Biology). The authors also thank Prof. A.F. Bakuzis's group (Federal University of Goiás, UFG, Institute of Physics, Goiânia, GO, Brazil) for the magnetization measurements and the LabMic-UFG for the TEM analysis.

This work was supported by São Paulo State Research Foundation-FAPESP (2010/14001-0), FAPDF, CAPES (CON-NANO), CNANO (UnB) and CNPq/MCT.

\section{References}

1. Khanna SS, Karjodkar FR (2006) Circulating immune complexes and trace elements (Copper, Iron and Selenium) as markers in oral precancer and cancer : a randomised, controlled clinical trial. Head Face Med 2: 33.

2. Zhang L, Yang X, Zhong LP, Zhou XJ, Pan HY, et al. (2009) Decreased expression of Annexin A1 correlates with pathologic differentiation grade in oral squamous cell carcinoma. J Oral Pathol Med 38: 362-370.

3. Kesting MR, Sudhoff H, Hasler RJ, Nieberler M, Pautke C, et al. (2009) Psoriasin (S100A7) up-regulation in oral squamous cell carcinoma and its relation to clinicopathologic features. Oral Oncol 45: 731-736.

4. Scully C, Bagan J (2009) Oral squamous cell carcinoma overview. Oral Onco 45: 301-308.

5. Maitland ML1, Schilsky RL (2011) Clinical trials in the era of personalized oncology. CA Cancer J Clin 61: 365-381.

6. Crozier E, Sumer BD (2010) Head and neck cancer. Med Clin North Am 94: 1031-1046.

7. Ruback MJ, Galbiatti AL, Arantes LM, Marucci GH, Russo A, et al. (2012) Clinical and epidemiological characteristics of patients in the head and neck surgery department of a university hospital. Sao Paulo Med J 130: 307-313.

8. Roberts C, Liyanage SH, Harry VN, Rockall AG (2011) Functional imaging for assessing tumor response in cancer of the cervix. Womens Health (Lond Engl) 7: 487-497.

9. Laurent S, Forge D, Port M, Roch A, Robic C, et al. (2008) Magnetic iron oxide nanoparticles: synthesis, stabilization, vectorization, physicochemical characterizations, and biological applications. Chem Rev 108: 2064-2110.

10. Teja AS, Koh PY (2009) Synthesis, properties, and applications of magnetic iron oxide nanoparticles. Prog Cryst Growth Charact Mater 55: 22-45.

11. Guedes MH, Sadeghiani N, Peixoto DLG, Coelho JP, Barbosa LLS, et al. (2005) Effects of AC magnetic field and carboxymethyldextran-coated magnetite nanoparticles on mice peritoneal cells. J Magn Magn Mater 293: 283-286.

12. Hilger I, Hergt R, Kaiser WA (2005) Use of magnetic nanoparticle heating in the treatment of breast cancer. IEE Proc Nanobiotechnol 152: 33-39.

13. Dennis CL, Jackson AJ, Borchers JA, Hoopes PJ, Strawbridge R, et al. (2009) Nearly complete regression of tumors via collective behavior of magnetic nanoparticles in hyperthermia. Nanotechnology 20: 395103.

14. Rosensweig RE (2002) Heating magnetic fluid with alternating magnetic field. J Magn Magn Mater 252: 370-374.

15. Hildebrandt B, Wust P, Ahlers O, Dieing A, Sreenivasa G, et al. (2002) The cellular and molecular basis of hyperthermia. Crit Rev Oncol Hematol 43: 3356.
16. Portilho FA, Estevanato LLC, Miranda-Vilela AL, Almeida-Santos MFM, de Oliveira-Cavalcanti CE, et al. (2011) Investigation of a magnetohyperthermia system efficacy. J Appl Phys 109: 07B307.

17. Balasenthil S, Saroja M, Ramachandran CR, Nagini S (2000) Of human and hamsters: comparative analysis of lipid peroxidation, glutathione, and glutathione-dependent enzymes during oral carcinogenesis. $\mathrm{Br} \mathrm{J}$ Ora Maxillofac Surg 38: 267-270.

18. Kornberg LJ, Villaret D, Popp M, Lui L, McLaren R, et al. (2005) Gene expression profiling in squamous cell carcinoma of the oral cavity shows abnormalities in several signaling pathways. Laryngoscope 115: 690-698.

19. Subapriya R, Bhuvaneswari V, Ramesh V, Nagini S (2005) Ethanolic leaf extract of neem (Azadirachta indica) inhibits buccal pouch carcinogenesis in hamsters. Cell Biochem Funct 23: 229-238.

20. Chandra Mohan KV, Hara Y, Abraham SK, Nagini S (2005) Comparative evaluation of the chemopreventive efficacy of green and black tea polyphenols in the hamster buccal pouch carcinogenesis model. Clin Biochem 38: 879-886.

21. de Freitas ER, Soares PR, Santos Rde P, dos Santos RL, da Silva JR, et al. (2008) In vitro biological activities of anionic gamma- $\mathrm{Fe}_{2} \mathrm{O}_{3}$ nanoparticles on human melanoma cells. J Nanosci Nanotechnol 8: 2385-2391.

22. Valois CR, Braz JM, Nunes ES, Vinolo MA, Lima EC, et al. (2010) The effect of DMSA-functionalized magnetic nanoparticles on transendothelial migration of monocytes in the murine lung via a beta2 integrin-dependent pathway. Biomaterials 31: 366-374.

23. Soler MA, Lima EC, Nunes ES, Silva FL, Oliveira AC, et al. (2011) Spectroscopic study of maghemite nanoparticles surface-grafted with DMSA. J Phys Chem A 115: $1003-1008$.

24. Cullity BD (1978) Elements of X-ray diffraction. (2ndedn), Canada: AddisonWesley.

25. Calmon MF, de Souza AT, Candido NM, Raposo MI, Taboga S, et al. (2012) A systematic study of transfection efficiency and cytotoxicity in HeLa cells using iron oxide nanoparticles prepared with organic and inorganic bases. Colloids Surf B Biointerfaces 100: 177-184.

26. Oliani SM, Lim LH, Christian HC, Pell K, Das AM, et al. (2001) Morphological alteration of peritoneal mast cells and macrophages in the mouse peritoneal cavity during the early phases of an allergic inflammatory reaction. Cell Biol Int 25: 795-803.

27. Zanatelli M, Silva DA, Shinohara FZ, Góes RM, Santos FC, et al. (2014) Actions of oestradiol and progesterone on the prostate in female gerbils: reversal of the histological effects of castration. Reprod Fertil Dev 26: 540-550.

28. Tartaj P, Morales MP, Veitemilhas-Verdaguer S, Gonzáles-Carreño T, Serna CJ (2003) The preparation of magnetic nanoparticles for applications in biomedicine. J Phys D Appl Phys 36: R81-R197.

29. Guan XH, Liu Q, Chen GH, Shang C (2005) Surface complexation of condensed phosphate to aluminum hydroxide: an ATR-FTIR spectroscopic investigation. $J$ Colloid Interface Sci 289: 319-327.

30. Parks GA (1965) The isoelectric points of solid oxide, solid hydroxides and aqueous hydroxocomplexe systems. Chem Rev 65: 177-198.

31. Cullity BD, Graham CD (1972) Introduction to magnetic materials. MA: AddisonWesley. Reading.

32. Viali WR, Alcantara GB, Sartoratto PPC, Soler MAG, Mosiniewicz-Szablewska $\mathrm{E}$, et al. (2010) Investigation of the molecular surface coating on the stability of insulating magnetic oils. J Phys Chem C 114: 179-188.

33. Shafi KVPM, Gedanken A, Prozorov R, Balogh J (1998) Sonochemical preparation and size-dependent properties of nanostructured $\mathrm{CoFe}_{2} \mathrm{O}_{4}$ particles. Chem Mater 10: 3445-3450.

34. Wu YN, Chen DH, Shi XY, Lian CC, Wang TY, et al. (2011) Cancer-cellspecific cytotoxicity of non-oxidized iron elements in iron core-gold shell NPs. Nanomedicine 7: 420-427.

35. Hirsch LR, Stafford RJ, Bankson JA, Sershen SR, Rivera B, et al. (2003) Nanoshell-mediated near-infrared thermal therapy of tumors under magnetic resonance guidance. Proc Natl Acad Sci U S A 100: 13549-13554.

36. Bernardi RJ, Lowery AR, Thompson PA, Blaney SM, West JL (2008) Immunonanoshells for targeted photothermal ablation in medulloblastoma and glioma: an in vitro evaluation using human cell lines. J Neurooncol 86: 165-172. 
Citation: Candido NM, Calmon MF, Taboga SR, Bonilha JL, dos Santos MC, et al. (2014) High Efficacy in Hyperthermia-associated with Polyphosphate Magnetic Nanoparticles for Oral Cancer Treatment. J Nanomed Nanotechnol 5: 206. doi:10.4172/2157-7439.1000206

37. Lemke AJ, Senfft von Pilsach MI, Lübbe A, Bergemann C, Riess H, et al. (2004) MRI after magnetic drug targeting in patients with advanced solid malignant tumors. Eur Radiol 14: 1949-1955.

38. Nel A, Xia T, Mädler L, Li N (2006) Toxic potential of materials at the nanolevel. Science 311: 622-627.

39. Lacava, ZGM, Morais PC (2004) Aplicações biomédicas de nanopartículas magnéticas. Parcerias Estratégicas/CGEE 18:73-85.

40. Barbugli PA, Siqueira-Moura MP, Espreafico EM, Tedesco AC (2010) In vitro phototoxicity of liposomes and nanocapsules containing chloroaluminum phthalocyanine on human melanoma cell line. J Nanosci Nanotechnol 10: 569573.

41. Souto EB, Wissing SA, Barbosa CM, Müller RH (2004) Evaluation of the physical stability of SLN and NLC before and after incorporation into hydroge formulations. Eur J Pharm Biopharm 58: 83-90.

42. Chithrani DB, Dunne M, Stewart J, Allen C, Jaffray DA (2010) Cellular uptake and transport of gold nanoparticles incorporated in a liposomal carrier. Nanomedicine 6: 161-169.

43. Soenen SJ, Nuytten N, De Meyer SF, De Smedt SC, De Cuyper M (2010) High intracellular iron oxide nanoparticle concentrations affect cellular cytoskeleton and focal adhesion kinase-mediated signaling. Small 6: 832-842.

44. Gerl R, Vaux DL (2005) Apoptosis in the development and treatment of cancer. Carcinogenesis 26: 263-270.

45. Ghobrial IM, Witzig TE, Adjei AA (2005) Targeting apoptosis pathways in cancer therapy. CA Cancer J Clin 55: 178-194.

46. Carneiro ML, Peixoto RC, Joanitti GA, Oliveira RG, Telles LA, et al. (2013) Antitumor effect and toxicity of free rhodium (II) citrate and rhodium (II) citrate-loaded maghemite nanoparticles in mice bearing breast cancer. J
Nanobiotechnology 11: 4

47. Maier-Hauff K, Rothe R, Scholz R, Gneveckow U, Wust P, et al. (2007) Intracranial thermotherapy using magnetic nanoparticles combined with external beam radiotherapy: results of a feasibility study on patients with glioblastoma multiforme. J Neurooncol 81: 53-60.

48. Balivada S, Rachakatla RS, Wang H, Samarakoon TN, Dani RK, et al. (2010) A/C magnetic hyperthermia of melanoma mediated by iron(0)/iron oxide core/ shell magnetic nanoparticles: a mouse study. BMC Cancer 10: 119.

49. Jones SK, Winter JG, Gray BN (2002) Treatment of experimental rabbit live tumours by selectively targeted hyperthermia. Int J Hyperthermia 18: 117-128.

50. Lacava LM, Garcia VAP, Küchelhaus SAS, Azevedo RB, Lacava ZGM, et al (2001) Magnetic resonance and light microscopy investigation of a dextran coated magnetic fluid. J Appl Phys 93: 7563-7565.

51. Estevanato LL, Lacava LM, Carvalho LC, Azevedo RB, Silva O, et al. (2012) Long-term biodistribution and biocompatibility investigation of dextran-coated magnetite nanoparticle using mice as the animal model. J Biomed Nanotechno 8: 301-308.

52. Wu W, He Q, Jiang C (2008) Magnetic iron oxide nanoparticles: synthesis and surface functionalization strategies. Nanoscale Res Lett 3: 397-415.

53. Garcia VAP, Lacava LM, Küchelhaus SAS, Da Silva MF, Morais PC, et al (2002) Magnetoliposomes: evaluation by citometry and micronucleus test. Eu Cells Mater 3:154-155.

54. Küchelhaus SAS, Garcia VAP, Lacava LM, Azevedo RB, Lacava ZGM et al. (2004) In vivo investigation of cobalt ferrit-based magnetic fluid and magnetoliposomes using morphological tests. J Magn Magn Mater 272: 2402 2403. 